\title{
Bedarfsplanung in der Augenheilkunde
}

CHRISTIAN KRAUTH, ANIKA BRÜMMER, NINA BUCHOLTZ, VOLKER E. AMELUNG

PD Dr. rer. pol. Christian Krauth, Dipl.-Volkswirt, ist Leiter des Fachbereichs Gesundheitsökonomie und Gesundheitspolitik am Institut für Epidemiologie, Sozialmedizin und Gesundheitssystemforschung der Medizinischen Hochschule Hannover,

Anika Brümmer, B.Sc ist Wissenschaftliche Mitarbeiterin im inav - privates Institut für angewandte Versorgungsforschung $\mathrm{GmbH}$,

Nina Bucholtz, Dipl. Psych. ist Doktorandin und Wissenschaftliche Mitarbeiterin an der Charité, Berlin,

Prof. Dr. oec. Volker Amelung ist Hochschullehrer am Institut für Epidemiologie, Sozialmedizin und Gesundheitssystemforschung der Medizinischen Hochschule Hannover und Vorstandsvorsitzender des Bundesverband Managed Care e. V.

Die Autoren danken der Deutschen Stiftung für chronische Kranke für die Förderung des Projektes.

\author{
Die Augenheilkunde wird aufgrund des \\ demographischen Wandels eine wichtige Rolle in der \\ ambulanten Versorgung einnehmen. Jeder Vierte \\ sucht mindestens einmal im Jahr einen Augenarzt auf \\ und schon heute sind $40 \%$ der Augenarztpatienten \\ 65 Jahre und älter. Dieser Trend wird sich durch die \\ weiter steigende Lebenserwartung noch verstärken, \\ da die Mehrheit der Augenerkrankungen erst \\ im Alter auftritt. Vor diesem Hintergrund ist die \\ Bedarfsplanung von besonderer Bedeutung. Auch wenn \\ bis 2020 kein substantieller Engpass an Augenärzten \\ existiert, müssen dennoch Strategien entwickelt \\ werden, die einen Versorgungsengpass in den wenig \\ attraktiven Planungsbereichen verhindern.
}

\section{Hintergrund: Situation der Augenheilkunde}

Die Fähigkeit zu sehen wird von vielen Menschen als selbstverständlich erachtet. Aber bereits leichte Sehbeeinträchtigungen führen zu einer Verringerung der Lebensqualität (Finger et al. 2011a). Personen mit visuellen Funktionsbeeinträchtigungen schätzen ihr physisches, emotionales und soziales Wohlbefinden signifikant schlechter ein als Personen ohne Beeinträchtigungen. Insbesondere die altersbedingte Makuladegeneration (AMD), der Grüne Star (Glaukom), der Graue Star (Katarkt) und die diabetische Retinopathie spielen in der Augenheilkunde eine besondere Rolle, nicht zuletzt weil sie die Hauptursache für Erblindungen darstellen (Finger et al. 2011b). Auch wenn das Behandlungsspektrum der Augenheilkunde »nur« einen kleinen Teil des menschlichen Körpers betrifft, leiden nach dem Arztreport der BARMER GEK (2010) ein Drittel der Versicherten an einer Augener- krankung bzw. Fehlsichtigkeit. Zudem sucht mindestens jeder Vierte einmal im Jahr einen Augenarzt auf. Damit liegt die Augenheilkunde mit 43,1 Behandlungsfällen pro 100 Personen an vierter Stelle der am häufigsten konsultierten Arztgruppen. Nur die Allgemeinmedizin, Innere Medizin und Gynäkologie weisen eine höhere Anzahl an Behandlungsfällen auf.

Trotz der hohen Anzahl an Behandlungsfällen repräsentieren die Augenärzte nur $2 \%$ der berufstätigen Ärzteschaft mit einer Gesamtanzahl von 6.805 berufstätigen Augenärzten (Bundesärztekammer 2011). Im Unterschied zu anderen fachärztlichen Bereichen befindet sich der überwiegende Anteil der Augenärzte mit 83,5\% im ambulanten Bereich. Verglichen mit allen berufstätigen Ärzten, von denen durchschnittlich $42,4 \%$ ambulant tätig sind, ist damit der Anteil ambulant tätiger Augenärzte fast doppelt so hoch. Diese Kennzahlen sind wenig verwunderlich, wenn man die Entwicklung des medizinisch-technischen 
Fortschritts in der Augenheilkunde betrachtet und den damit einhergehenden Verbesserungen in Diagnostik und Therapie. Beispielsweise ist die Katarakt weltweit für $50 \%$ der Erblindungen verantwortlich, während in Europa und den USA durch gute operative Behandlungen die Erblindungsrate durch eine Katarakt nur bei 5\% liegt (Resnikoff et al. 2004, WHO 2004). Darüber hinaus können mittlerweile durch neu entwickelte Medikamente und Therapien wie die intravitreale operative Medikamentenapplikation (IVOM) die feuchte AMD, die in Deutschland die häufigste Ursache für Erblindungen darstellt, behandelt werden. Aber auch bei anderen Indikationen, wie bei Makulaödemen der diabetischen Retinopathie oder Venenverschlüssen wird die IVOM angewendet. Der medizinische Fortschritt ermöglicht damit eine Verschiebung der Behandlung vom stationären zum ambulanten Sektor, wodurch der hohe Anteil an ambulant tätigen Augenärzten erklärbar wird.

$\mathrm{Zu}$ den großen zukünftigen Herausforderungen in der Augenheilkunde gehören der demographische Wandel und die zunehmende Lebenserwartung. Die Mehrheit der Augenerkrankungen treten erst im Alter auf, wodurch zukünftig mit einem Anstieg der in Anspruch genommenen augenärztlichen Leistungen gerechnet wird: Während in 1997 nur 43\% der Patienten in einer Augenarztpraxis über 60 Jahre alt waren, sind es aktuell fast 50\% (KBV 2011). Zudem sucht bereits jeder Zweite in der

\section{Der augenärztliche Fachbereich entwickelt sich aufgrund des steigenden Lebensalters zu einem Grundversorger.}

\section{Theoretische Grundlagen zur Bedarfsplanung}

In 1993 wurde die Bedarfsplanung des Gemeinsamen Bundesausschusses (GBA) mit dem Gesundheitsstrukturgesetz in das SGB V eingeführt. Ziel ist es, die Versorgung der Bevölkerung mit medizinischen Leistungen im ambulanten wie auch stationären Sektor sicherzustellen. Für den Bereich der Augenheilkunde spielt die ambulante Bedarfsplanung die zentrale Rolle. Zur Bestimmung des Bedarfs wird die Relation der Einwohner je Vertragsarzt herangezogen, wobei

Altersgruppe der 70- bis 84-Jährigen jährlich einen Augenarzt auf. Prognostiziert wird, dass sich die Anzahl der an Makuladegeneration bzw. Glaukom Erkrankten bis 2050 um 169\% bzw. $72 \%$ erhöhen wird (Fritz Beske Institut 2009). Auch das Zentralinstitut für die kassenärztliche Versorgung (ZI) ermittelte, dass die medizinischen Leistungen durch Internisten, Urologen, Hausärzte und Augenärzte am stärksten zunehmen werden (Schallock et al. 2009). Vor die- die Verhältniszahlen nach Facharztgruppen differenziert werden und sich zwischen urbanen und ländlichen Regionen unterscheiden (s. Tab 1).

Erstellt wird der Bedarfsplan von dem Landesausschuss der Ärzte und Krankenkassen nach $\mathbb{\int} 99$ SGB V. Dafür wird die Relation von Einwohner zu Arzt für jeden Planungsbezirk berechnet und mit den allgemein festgelegten Verhältniszahlen verglichen. Liegt der Versorgungsgrad um mehr als 10\% höher als der bundesweit festgelegte Versorgungsgrad, ist der Planungsbezirk überversorgt ( $\mathbb{S} 101$ SGB V). Als unterversorgt gilt ein Planungsbezirk, wenn der bundesweit festgelegte Versorgungsgrad um mehr als 50\% unterschritten wird ( 100 SGB V). Seit 2010 fließt ein Demografiefaktor in die Bedarfsplanung des G-BA ein, um den erhöhten Versorgungsbedarf eines Planungsbezirks mit einer relativ älteren Bevölkerung (gegenüber dem Bundesdurchschnitt) zu berücksichtigen.

\section{Bedarfsplanung der Kassen- ärztlichen Bundesvereinigung in der Augenheilkunde}

Die Kassenärztliche Bundesvereinigung (KBV) legt auf der Basis der Bedarfsplanung des G-BA die Anzahl der offenen Planungsbereiche, bei insgesamt 395 Planungsbereichen in Deutschland, fest. Unter offenen Planungsbereichen versteht man solche Bereiche, die nicht überversorgt (Versorgungsgrad $<110 \%$ ) sind und in denen sich Vertragsärzte frei niederlassen können.

In der Augenheilkunde sinkt die Anzahl der freien Arztsitze wie auch die Anzahl der offenen Planungsbereiche stetig. Im Jahr 2000 gab es 103 offene Planungsbereiche mit 150 freien Arztsitzen, während es in 2011 nur noch 53 offene Planungsbereiche mit 93 freien Arztsitzen waren (KBV 2011). Dabei liegt der höchste Anteil an freien Arztsitzen in Bayern (18), Niedersachsen (19) und Rheinland-Pfalz (15) und dement- 


\begin{tabular}{|l|c|l|c|}
\hline \multicolumn{1}{|c|}{ Tabelle 2: Planungsbereich mit höchsten bzw. niedrigsten Versorgungsgrad (Quelle: Deutscher Bundestag 2011) } \\
\hline \multicolumn{1}{|c|}{ Planungsbereich } & Versorgungsgrad max. & Planungsbereich & Versorgungsgrad min. \\
\hline Neubrandenburg, Stadt/Mecklenburg-Strelitz & 227,9 & Odenwaldkreis & 47,7 \\
\hline Weiden i.d. Opf., Stadt/Neustadt a.d. Waldnaab & 216,2 & Mittlerer Erzgebirgskreis & 55,7 \\
\hline Prignitz & 214,2 & Mainz-Bingen & 60,2 \\
\hline Osterode am Harz & 198,7 & Fürth & 63,7 \\
\hline Würzburg, Stadt & 198,5 & Bad Doberan & 65,9 \\
\hline Uckermark & 193,5 & Peine & 67,3 \\
\hline Greifswald, Hansestadt/Ostvorpommern & 188,6 & Enzkreis & 68,5 \\
\hline Miesbach & 185,1 & Gifhorn & 74,5 \\
\hline Stralsund, Hansestadt/Nordvorpommern & 183,9 & Schwandorf & 79,3 \\
\hline Löbau-Zittau & 182,5 & Gotha & 79,8 \\
\hline
\end{tabular}

sprechend befinden sich auch in diesen KV-Bezirken die meisten offenen Planungsbereiche.

Die kontinuierliche Abnahme von offenen Planungsbereichen führt dazu, dass Augenärzte auf bisher noch nicht gesperrte Planungsbereiche ausweichen. Damit nähert sich die räumliche Verteilung der augenärztlichen Versorgung dem Bedarf an, wie er in den Richtlinien des G-BA ausgewiesen wird. Trotz dessen bestehen aber zwischen den Planungsbereichen große Versorgungsunterschiede. Dies bestätigen auch aktuelle Zahlen der KBV, die von der Bundesregierung angefordert wurden (Deutscher Bundestag 2011). Es zeigt sich, dass der Versorgungsgrad mit augenärztlichen Leistungen in den 395 Planungsbereichen zwischen 48\% (Odenwaldkreis) und 228\% (Neubrandenburg) stark schwankt. Darüber hinaus weisen 342 der 395 Planungsbereiche einen Versorgungsgrad von über $110 \%$ auf, was auf eine Überversorgung schließen lässt. Lediglich 8 Planungsbereiche liegen unter einem Versorgungsgrad von $75 \%$ und ein Planungsbereich ist mit einem Versorgungsgrad von unter $50 \%$ gemäß der Bedarfsrichtlinie des G-BA von Unterversorgung betroffen (siehe Tab. 2). Um in allen Planungsbereichen mindestens die Normversorgung von $100 \%$ zu erreichen, müssen bundesweit 44 Arztsitze (in 27 Planungsbereichen) besetzt werden. Bis zum Erreichen der Überversorgung (Versorgung $>110 \%$ ) fehlen sogar 93 Arztsitze in 53 Planungsbereichen.

Besonders auffällig ist der hohe Versorgungsgrad vieler Planungsbereiche in den neuen Bundesländern (sechs der zehn Planungsbereiche mit dem höchsten Versorgungsgrad). Dieser Effekt lässt sich teilweise durch den Bevölkerungsrückgang nach der Wiedervereinigung erklären, wodurch bei gleichbleibender Anzahl an Augenärzten eine geringere Einwohner-Arzt-Relation entsteht. Allerdings muss berücksichtigt werden, dass auch drei der zehn Planungsbereiche mit den geringsten Versorgungsgraden in den neuen Bundesländern liegen. Neben dem deutlichen Bevölkerungsrückgang sind die neuen Bundesländer auch durch eine starke Alterung der Bevölkerung gekennzeichnet, da der Bevölkerungsrückgang überwiegend durch die Abwanderung junger Menschen in die alten Bundesländer erfolgte. Die demografischen Faktoren sollten in der Bedarfsplanung angemessen berücksichtigt werden, insbesondere der höhere Versorgungsbedarf älterer gegenüber jüngerer Menschen. Dies würde den bisher ausgewiesenen Versorgungsgrad in den neuen Bundesländern teilweise deutlich senken. Der bisher eingeführte Demografiefaktor scheint diesen Aspekt nicht vollständig auszugleichen.

\section{Prognosen des zukünftigen augenärztlichen Bedarfs}

\subsection{Einflussfaktoren des Angebots an und der Nachfrage nach augenärztlichen Leistungen}

Um prognostische Aussagen über die zukünftige augenärztliche Versorgung zu erheben, müssen verschiedene Einflussfaktoren des Angebots an und der Nachfrage nach augenärztlichen Leistungen berücksichtigt werden. $\mathrm{Zu}$ den Determinanten, die das augenärztliche Angebot bedingen, gehören:
- Altersstruktur der Ärzte (Durchschnittsalter der Vertragsärzte, Anteil der Ärzte mit 55, 60 bzw. 65 Jahren)

- Nachfolgeregelung in überversorgten Gebieten

- "Feminisierung der Medizin «

- Trend zu kürzeren Arbeitszeiten der Ärzte

- Rekrutierungspotenzial des augenärztlichen Nachwuchs (Reglementierung und Honorierung)

Die Altersstruktur der Ärzte gehört zu den ausschlaggebenden Faktoren für das zukünftige augenärztliche Angebot, da an der Altersstruktur der Ersatzbedarf an Augenärzten in den nächsten Jahren abgelesen werden kann. Derzeit liegt das Durchschnittsalter der Augenärzte bei 52,2 Jahren und stieg damit in den letzten 15 Jahren um 4,7 Jahre an (Wille 2011). Insgesamt haben 34\% der berufstätigen Augenärzte bereits das 55 . Lebensjahr überschritten.

Die Nachfolgeregelung in überversorgten Gebieten besitzt ebenso einen entscheidenden Einfluss auf den Ersatzbedarf. Bisher kann jeder Vertragsarzt, der aus Altersgründen seine Praxis aufgibt, seine KV mit der Ausschreibung des Vertragsarztsitzes beauftragen. Dies ist auch in überversorgten Gebieten möglich. Durch die Regelung wird die Neubesetzung von Arztsitzen in weniger begehrten Planungsbereichen erschwert, die tendenziell von Unterversorgung betroffen sind. Die Erfahrungen zeigen, dass Vertragsärzte auf nicht gesperrte Planungsbereiche ausweichen, wenn Niederlassungsmöglichkeiten in attraktiven Regionen gesperrt sind.

Zudem ist ein deutlicher Trend zur »Feminisierung der Medizin« in der 


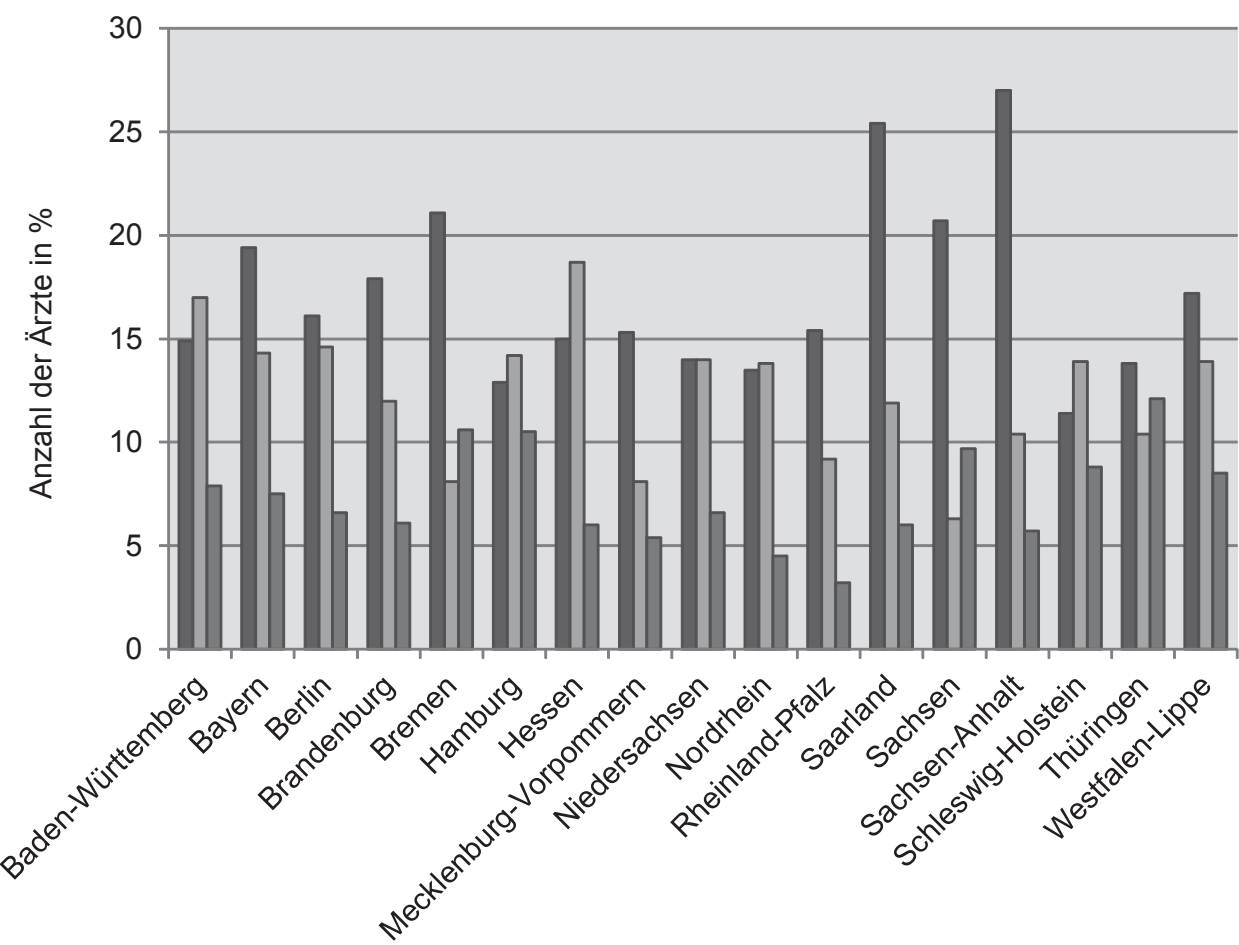

- Ärzte ab 55 bis 59 Jahre $\square$ Ärzte ab 60 bis 64 Jahre $\square$ Ärzte ab 65 Jahren und älter

Abbildung 1: Augenärzte über 55, 60 und 65 Jahren, die an der kassenärztlichen Versorgung beteiligt sind (Quelle: KBV 2011)

Ärzteschaft zu beobachten. In den letzten 18 Jahren stieg der Anteil der Ärztinnen an den Vertragsärzten von $29 \%$ auf $36 \%$ an. Dieser Trend wird sich auch zukünftig mit aktuell 61\% weiblichen Medizinstudenten fortsetzen (Destatis 2011). Diese Entwicklung wirkt sich auf das Angebot an Versorgungsleistungen aus, da Ärztinnen (zumindest derzeit noch) durchschnittlich häufiger in Teilzeit beschäftigt sind als ihre männlichen Kollegen. Aber auch insgesamt zeichnet sich der Trend hin zu kürzeren Arbeitszeiten bei jüngeren Ärzten ab. So liegt die Wochenarbeitszeit von Ärzten bis 35 Jahren bei ca. 46 Stunden, während die durchschnittliche Wochenarbeitszeit aller Ärzte ca. 49 Stunden beträgt (Wille 2010).

Entscheidenden Einfluss auf die Nachfrage nach augenärztlichen Leistungen besitzt die demografische Entwicklung, da viele Augenerkrankungen erst im Alter auftreten. Während die demografische Alterung zu einer steigenden Inanspruchnahme von ärztlichen Leistungen führt, reduziert sich die Nachfrage ceteris paribus bei sinkender Bevölkerung. Der Nettoeffekt ist entscheidend, ob mehr oder weniger augenärztliche Leistungen zukünftig in Anspruch genommen werden.

\subsection{Bedarfsanalyse auf Basis prognostizierter Arztzahlen am Beispiel von Niedersachsen}

Die KV Niedersachsen (KVN) führte 2007 eine Hochrechnung der Arztzahlen bis 2020 durch. Dafür wurde ausgehend vom aktuellen Arztbestand die Entwicklung der Zu- und Abgänge an Augenärzte prognostiziert. Für die Ermittlung der Zugänge wurden die Facharztzulassungen von 2002 bis 2006 verwendet und anschließend für die Jahre 2008 bis 2020 geschätzt. Zur Bestimmung der Abgänge an Augenärzten wurde das Alter herangezogen und anhand von zwei Szenarien simuliert mit: (1) Altersabgänge erfolgen mit 60 Jahren und (2) Altersabgänge erfolgen erst mit 68 Jahren. Darüber hinaus berücksichtigte die KVN einen Bevölkerungsrückgang bis 2020 um ca. 3,1\% bzw. 240.000 Einwohner verglichen zu 2008.

Die Bedarfsanalyse der KVN für den Bereich Augenheilkunde zeigt, dass die prognostizierten Altersabgänge mit 60 Jahren nicht vollständig durch die erwarteten Zugänge ausgeglichen werden können. So sinkt im Szenario 1 (Altersabgänge mit 60 Jahren) der Arztbestand bis 2020 um ca. 8,2\% bzw. 38 Augenärzte, allerdings lässt sich auch mit dem geringeren Arztbestand eine Normversorgung von $100 \%$ aufrechterhalten (vorausgesetzt es gelingt eine erfolgreiche Umverteilung von hochversorgten zu geringversorgten Planungsbereichen in Niedersachsen). Damit entsprechen auch die notwendigen Zulassungen mit 232,3 Augenärzten den kumulierten prognostizierten Zugängen (234,0 Augenärzte) bis 2020, um einen Versorgungsgrad von $100 \%$ zu sichern. Zur Vermeidung von Unterversorgung (Versorgungsgrad $<50 \%$ ) werden lediglich 31,5 neue Augenärzte benötigt.

Im Szenario 2 mit Altersabgängen ab 68 Jahren stellt sich die Versorgungssituation erheblich besser dar. In diesem Fall liegen die Zugänge an Augenärzten mit 234 deutlich über den Altersabgängen an Augenärzten von 146 . Nur $50 \%$ der erwarteten Facharztabsolventen (113 von 234) müssen bis 2020 zur kassenärztlichen Versorgung zugelassen werden, um die Normversorgung von 100\% in allen Planungsbereichen aufrechtzuerhalten. Soll nur eine Unterversorgung vermieden werden, müssen lediglich 3 neue Augenärzte zugelassen werden.

Bei der Bewertung der Bedarfsprognose der KVN muss allerdings eine differenzierte Betrachtung erfolgen. Auf dem ersten Blick scheint die Versorgung in Niedersachsen bis 2020 ge- 
Tabelle 3: Bevölkerungsprognose (Quelle: Statistischen Bundesamtes 2009)

\begin{tabular}{|l|c|c|c|c|c|c|}
\hline Altersgruppe & \multicolumn{2}{|c|}{$\mathbf{2 0 0 8}$} & \multicolumn{2}{|c|}{$\mathbf{2 0 2 0}$} & \multicolumn{2}{|c|}{$\mathbf{2 0 3 0}$} \\
\hline & Bevölkerung in 1.000 & $\%$ & Bevölkerung in 1.000 & $\%$ & Bevölkerung in 1.000 & $\%$ \\
\hline Gesamt & 82.002 & & 79.914 & & 77.350 \\
\hline Unter 20 Jahre & 15.619 & 19,0 & 13.624 & 17,0 & 12.927 & 16,7 \\
\hline 20 bis unter 60 Jahre & 45.426 & 55,4 & 41.743 & 52,2 & 35.955 & 46,5 \\
\hline 60 Jahre und älter & 20.958 & 25,6 & 24.547 & 30,7 & 28.469 & 36,8 \\
\hline
\end{tabular}

sichert zu sein, jedoch gibt es regional deutliche Versorgungsunterschiede. So wird derzeit bereits in 6 von $44 \mathrm{Pla}-$ nungsbereichen in Niedersachsen nicht die Normversorgung von $100 \%$ erreicht und weitere 2 Planungsbereiche liegen sogar unter einem Versorgungsgrad von $75 \%$. Im Gegensatz dazu gibt es aber auch 11 Planungsbezirke (also 25\% aller Planungsbereiche der KVN), die einen Versorgungsgrad zwischen $125 \%$ und $200 \%$ aufzeigen. Zudem variiert der Ersatzbedarf in den Planungsbereichen stark durch die unterschiedliche regionale Altersstruktur der Augenärzte. Darüber hinaus liegt hier im Wesentlichen eine angebotsorientierte Prognose vor. Bei der Nachfrage nach augenärztlichen Leistungen wurde nur der Bevölkerungsrückgang berücksichtigt, aber nicht die zunehmende Alterung der Bevölkerung. Um den demographischen Wandel angemessen zu berücksichtigen, wurden eigene Hochrechnungen für die zukünftige augenärztliche Versorgung durchgeführt. Der demographische Wandel spielt insofern eine besondere Rolle, da der überwiegende Anteil an Augenerkrankungen erst im Alter auftritt und somit durch die Zunahme der älteren Bevölkerungsteile auch eine Ausweitung der in Anspruch genommenen augenärztlichen Leistungen zu vermuten ist.

\subsection{Bevölkerungsprognose und Hochrechnung der augenärztlichen Patientenzahlen bis 2030}

Zur Abschätzung der zukünftigen Nachfrage nach augenärztlichen Leistungen ist eine Prognose über die Bevölkerungsentwicklung und einer darauf aufbauenden Hochrechnung der augenärztlichen Patienten notwendig.

Für die Bevölkerungsprognose konnte auf Daten des Statistischen Bundesamtes (2009) zugegriffen werden. Das Statistische Bundesamt hat in seiner mittlerweile 12. Koordinierten Bevölkerungsberechnung verschiedene Varianten mit den demografischen Faktoren Geburtenrate, Mortalität und Migration gebildet. Für die nachfolgenden Analysen werden die mittleren Prognosevarianten der Bevölkerungsberechnung herangezogen. Wie aus der Tabelle 3 ersichtlich wird, steigt der Anteil der über 60-Jährigen von $25,6 \%$ (2008) auf $36.8 \%$ (2030). Damit ist eine Zunahme um 7,5 Mio. der älteren Bevölkerungsgruppe $a b$ 60 Jahren zu erwarten bei gleichzeitiger Verringerung der Gesamtbevölkerung um 4,7 Mio. bis zum Jahr 2030.

Auf der Basis der Bevölkerungsprognose können nun im zweiten Schritt die Patientenzahlen in der Augenheilkunde bis 2030 prognostiziert werden. Dafür wird zunächst analysiert, wie viele $\mathrm{Pa}$ tienten derzeit pro Jahr einen Augenarzt aufsuchen. Diese Berechnungen beruhen auf Daten der KBV (2011). Demzufolge konsultieren ca. 50\% der über 75-jährigen GKV-Versicherten mindestens einmal im Jahr einen Augenarzt, während es in der Altersgruppe der 15 bis 50-jährigen GKV Versicherten lediglich 10 bis $20 \%$ sind. Wie vorab vermutet, ist die Leistungsinanspruchnahme stark abhängig vom Alter des Patienten (siehe Abb. 2).

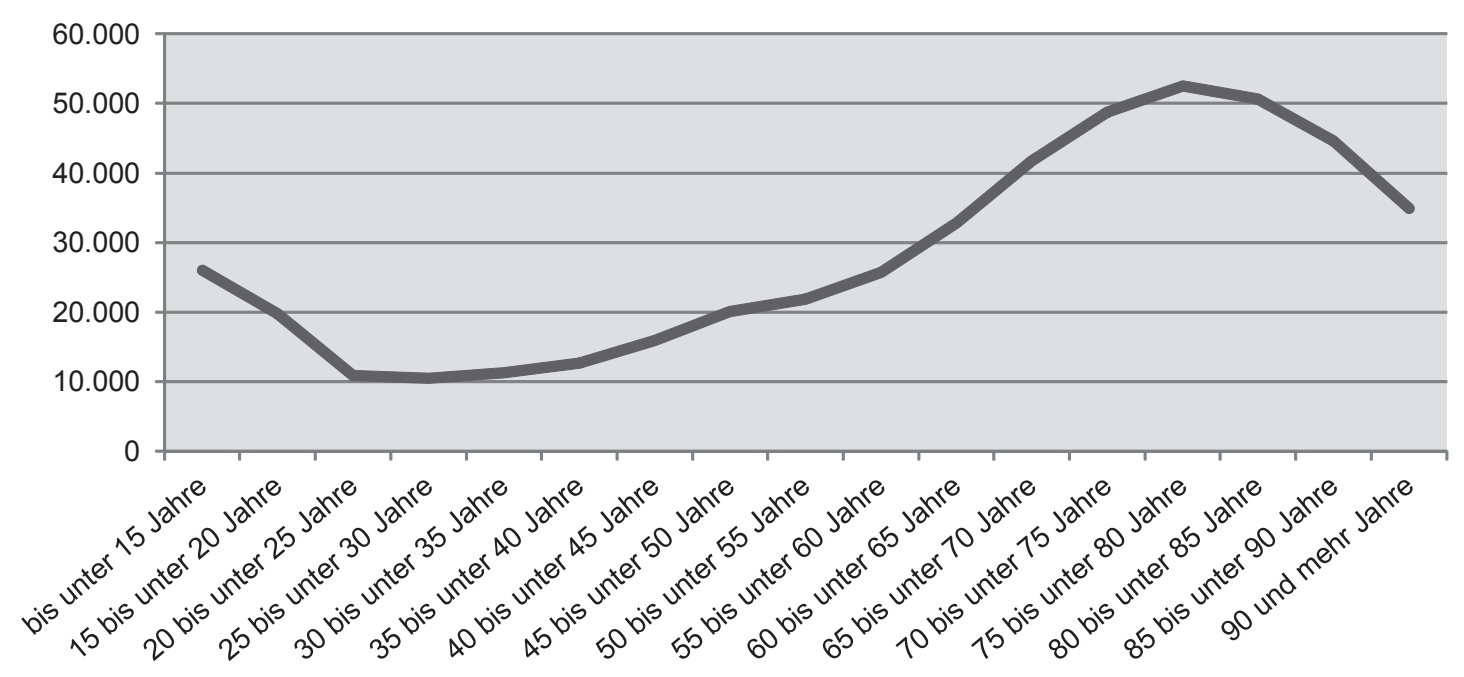

Abbildung 2: Patienten beim Augenarzt je 100.000 Versicherte der Altersgruppe in 2010 (Quelle: KBV 2011) 


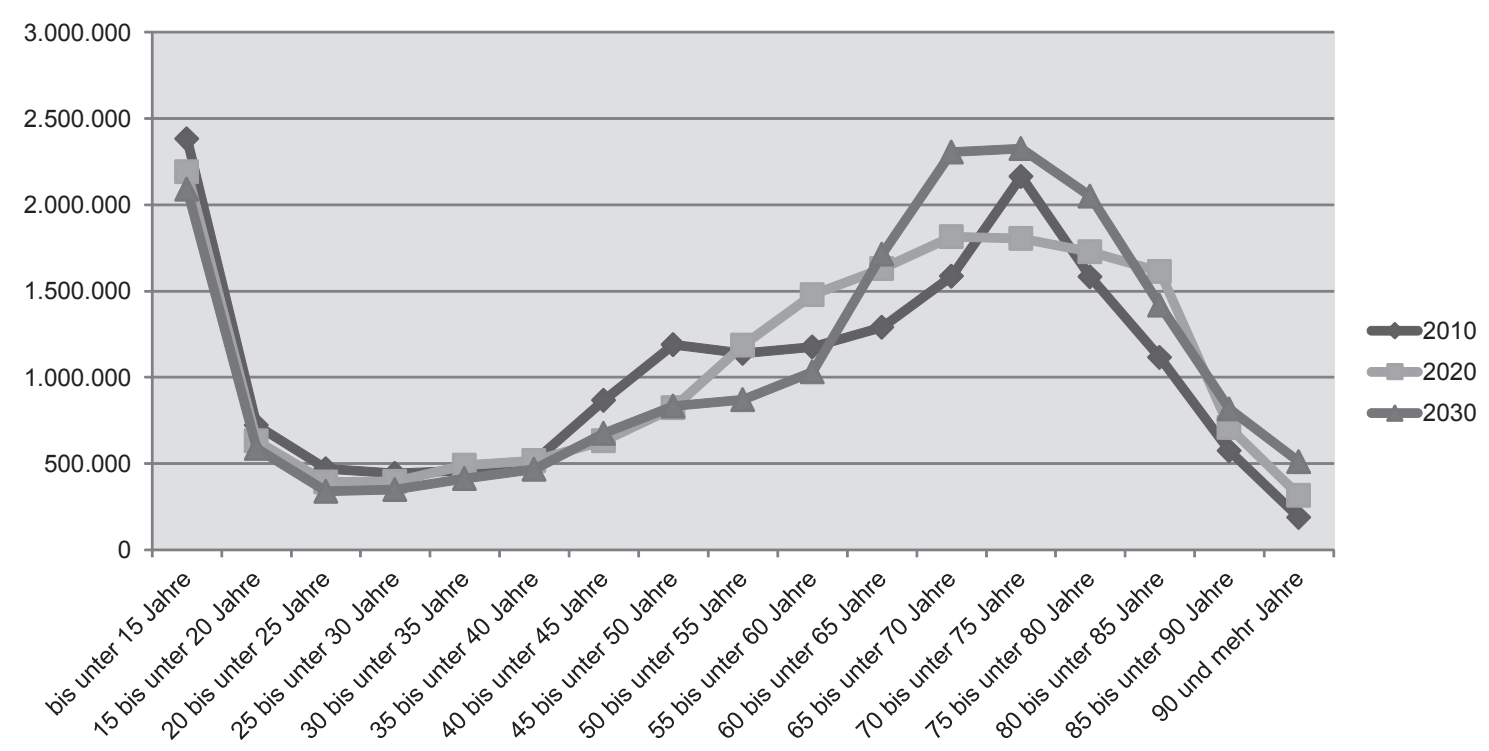

Abbildung 3: Prognose der Patientenzahlen in der Augenheilkunde (eigene Darstellung)

Nachdem die aktuelle Patientenzahl, untergliedert in Altersgruppen, ermittelt wurde, erfolgt die Hochrechnung der zukünftigen Patientenzahlen gemäß der folgenden Gleichung:

$P_{t}=\sum_{i} P_{i}=\sum_{i} a_{2010, i} \cdot G_{2010, i} \cdot\left(B_{i} / B_{2010, i}\right)$

mit

$P_{t} \quad$ Patientenanzahl im Jahr $\mathrm{t}(\mathrm{t}=2010$ bis 2030)

$P_{t i} \quad$ Patientenanzahl der Altersgruppe i im Jahr $\mathrm{t}$ (Altersgruppen 0-15/ 15-20/ 20-25/ ... / 85-90 und $>90$ Jahre)

$a_{2010, i}$ Anteil der GKV-Versicherten der Altersgruppe i im Basisjahr 2010, die einen Augenarzt konsultiert haben (ausgedrückt als Anzahl der GKV-Versicherten der Altersgruppe i, die 2010 einen Augenarzt konsultierten, je 100.000 GKVVersicherte der Altersgruppe i)

$G_{2010}$ Anzahl GKV-Versicherte im Jahr 2010

$B_{t} \quad$ Anzahl der Personen der Altersgruppe i im Jahr t

Um zukünftige Patientenzahlen der GKV zu prognostizieren, wird von den aktuellen altersspezifischen Patientenzahlen beim Augenarzt $\left(a_{2010, i} \cdot G_{2010, i}\right)$ ausgegangen und mit der zukünftigen Altersstruktur der Bevölkerung in Deutschland $\left(B_{t, i} / \mathrm{B}_{2010, i}\right)$ hochgerechnet. Dabei wird angenommen, dass die altersspezifische Inanspruchnahme im Zeitablauf konstant bleibt $\left(a_{2010, i}=a_{2020, i}\right.$ $=a_{2030, i}$. .

Die durchgeführte Analyse gibt nun Aufschluss über die altersdifferenzierten
Patientenzahlen $P_{t i}$ für die Jahre 2010, 2020 und 2030 (siehe Abb. 3). Zwei Entwicklungen werden dabei in der Prognose widergespiegelt: (1) Die Verringerung der Gesamtbevölkerung von 82,0 Mio. (2010) über 79,9 Mio. (2020) auf 77,4 Mio. (2030) bei (2) gleichzeitigem Anstieg der Bevölkerungsgruppe der über 65-Jährigen von 16,8 Mio. (2010) über 18,7 Mio. (2020) auf 22,3 Mio. (2030).

Insgesamt wird die Patientenzahl in der Augenheilkunde kontinuierlich von 17,9 Mio. in 2010 auf 18,8 Mio. in 2020 bzw. 19,0 Mio. in 2030 zunehmen, was einem Anstieg von 5,2\% bis 6,4\% entspricht. Demzufolge kann konstatiert werden, dass der Alterungseffekt (Anstieg der über 65-jährigen von 2008 bis 2030 um 5,5 Mio.) den Bevölkerungseffekt (Abnahme der Gesamtbevölkerung von 2008 bis 2030 um 4,6 Mio.) bei der Nachfrage nach augenärztlichen Leistungen übersteigt. Folglich wird die Nachfrage nach augenärztlichen Leistungen stetig anwachsen.

Die Ergebnisse werden durch alternative Prognosen zum zukünftigen Bedarf an Augenärzten gestützt. Beispielsweise geht der Berufsverband der Augenärzte (BVA) von einem Anstieg nach augenärztlichen Leistungen in Höhe von 10\% bis zum Jahr 2030 aus. Wille (2010) führte eine Studie zur Entwicklung der Arztkontakte in Baden-Württemberg und Bayern durch. Prognostiziert wurde eine Zunahme der augenärztlichen ArztPatienten-Kontakte bis 2030 in Bayern

um 14,5 bis $15,5 \%$ und in Baden-Württemberg um 16 bis 17\% (Wille 2010, S. 67). Die unterschiedlichen Ergebnisse zwischen den Studien sind auf mehrere Einflussfaktoren zurückzuführen. Die Studie von Wille ist nur eingeschränkt auf Gesamt-Deutschland übertragbar, da zum einen der Bevölkerungsrückgang in Bayern und Baden-Württemberg deutlich geringer ausfällt als im Bundesdurchschnitt und zum anderen die Lebenserwartung in Baden-Württemberg über dem Bundesdurchschnitt liegt. Insofern wird die Nachfrage nach Augenarztleistungen (bezogen auf Deutschland insgesamt) in der Studie von Wille überschätzt. Umgekehrt ist es plausibel, dass die Arztkontakte (Wille-Studie) stärker ansteigen als die Patientenzahlen (vorliegende Hochrechnung), da nicht nur ein größerer Anteil älterer Versicherter (gegenüber jüngeren Versicherten) den Augenarzt konsultiert, sondern ältere Patienten (die einen Augenarzt

Ein Nachfrageanstieg in Deutschland von $10-12 \%$
scheint plausibel.

aufsuchen) auch mehr Arztkontakte als jüngere Patienten haben. Insofern wird die Nachfrage in der vorliegenden Studie unterschätzt. Insgesamt erscheint ein Nachfrageanstieg in Deutschland von $10-12 \%$ plausibel (bei einer Bandbreite von $6-15 \%)$. 
Tabelle 4: Veränderung des Versorgungsniveaus 2020 gegenüber 2008 - Szenarien in Abhängigkeit von Veränderungen der Nachfrage, der Arztanzahl und der Wochenarbeitszeit (eigene Darstellung)

\begin{tabular}{|c|c|c|c|c|}
\hline \multicolumn{5}{|c|}{ Szenarien der zukünftigen augenärztlichen Versorgung bis 2020} \\
\hline \multirow{7}{*}{ 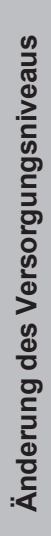 } & Angebot & \multicolumn{3}{|c|}{ Nachfrageanstieg } \\
\hline & $\begin{array}{l}\text { Altersabgänge } 60 \\
\text { (Anzahl Augenärzte } \quad-7 \% \text { ) }\end{array}$ & & & \\
\hline & Konstante Wochenarbeitszeit & $-10 \%$ & $-11 \%$ & $-13 \%$ \\
\hline & Sinkende Wochenarbeitszeit (5\%) & $-14 \%$ & $-16 \%$ & $-17 \%$ \\
\hline & $\begin{array}{l}\text { Altersabgänge } 68 \\
\text { (Anzahl Augenärzte +19\%) }\end{array}$ & & & \\
\hline & Konstante Wochenarbeitszeit & $+16 \%$ & $+13 \%$ & $+11 \%$ \\
\hline & Sinkende Wochenarbeitszeit $(5 \%)$ & $+10 \%$ & $+8 \%$ & $+6 \%$ \\
\hline
\end{tabular}

\section{Szenarien der zukünftigen augenärztlichen Versorgung}

Nachdem das Angebot an und die Nachfrage nach augenärztlichen Leistungen ermittelt wurden, können diese Daten in verschiedene Szenarien zur zukünftigen augenärztlichen Versorgung zusammengeführt werden. Die Szenarien werden nur bis zum Jahr 2020 fortgeschrieben, da die Ergebnisse für das augenärztliche Angebot ebenfalls nur bis 2020 belastbar vorliegen. Für die Szenarien wird auf die Prognosen zum Arztangebot der KV Niedersachsen zugegriffen und auf Deutschland insgesamt übertragen.

Als Determinanten für das augenärztliche Angebot werden die Altersabgänge mit 60 bzw. 68 Jahren entsprechend der KVN-Prognose herangezogen: bei Altersabgängen mit 60 Jahren muss mit einer Verminderung des Angebots von ca. 7\% gerechnet werden, während das Arztangebot bei Altersabgängen mit 68 Jahren um 19\% ansteigt. Zusätzlich wird in einer Sensitivitätsanalyse angenommen, dass die durchschnittliche Arbeitszeit der Ärzte bis 2020 um 5\% sinkt. Für die Nachfrage nach augenärztlichen Leistungen werden die eigenen Berechnungen zur bundesweiten Hochrechnung der Patientenzahlen bis 2020 und die Analysedaten der WilleStudie einbezogen. Dementsprechend liegt der erwartete Anstieg der nachgefragten Leistungen bei $5 \%$ mit einer Bandbreite von 3 bis 7\% (siehe Tab. 4).

Die verschiedenen Szenarien zeigen, dass die Altersabgänge der Augenärzte das Versorgungsniveau bis 2020 erheblich beeinflussen. Die extremen Szenarien zeigen folgendes Bild:

Szenario 1: Im ersten extremen Szenario wird die Änderung des Versorgungsniveaus gekennzeichnet durch Altersabgänge mit 60 Jahren (Reduktion der Arztanzahl um ca. 7\%) und eine Minderung der Wochenarbeitszeit der niedergelassenen Augenärzte um 5\% bei einem Nachfrageanstieg um 7\%. In diesem Fall würde das Versorgungsniveau um ca. $17 \%$ verglichen zur aktuellen Versorgungssituation schrumpfen. Damit sinkt der durchschnittliche Versorgungsgrad in den Planungsbereichen auf etwa $83 \%$. Eine flächendeckende Normversorgung ist dann auch rechnerisch wenn also ein Ausgleich von über- und unterversorgten Planungsbereichen erfolgt - nicht mehr realisiert.

Szenario 2: Für das zweite extreme Szenario erfolgen die Altersabgänge

Bis 2020 ist kein substantieller Engpass in der Augenheilkunde zu erwarten.

erst mit 68 Jahren (Anstieg der Arztanzahl um ca. 19\%), die durchschnittliche Wochenarbeitszeit bleibt konstant und die Nachfrage nimmt um 3\% zu. Diese Kombination führt zu einem Anstieg des Versorgungsniveaus in 2020 um ca. $16 \%$. Dadurch wird in allen Planungs- bezirken ein durchschnittlicher Versorgungsgrad von $120 \%$ erreicht.

Szenario 3: Das Szenario 3 stellt eine mittlere Variante der vorangegangenen Szenarien dar. Hierfür wird mit einem Anstieg des Arztangebotes von 6\% (der sich aus dem Mittelwert der extremen Szenarien Rückgang um 7\% und Anstieg um 19\% erklärt), einer konstanten durchschnittlichen Wochenarbeitszeit der niedergelassenen Augenärzte und einem Anstieg der Nachfrage um 5\% gerechnet. In diesem Szenario kann das derzeitige Versorgungsniveau auch in 2020 sichergestellt werden.

In den Szenarien konnte das Versorgungsniveau nur durch eine Verlängerung der Lebensarbeitszeit der Augenärzte aufrechterhalten werden. Zwar zeigen sich bereits erste Anzeichen dafür, dass die niedergelassenen Ärzte länger praktizieren. Allerdings muss beobachtet werden, ob zusätzliche Anreize zur Verlängerung der Lebensarbeitszeit notwendig sind. Darüber hinaus muss die regionale Bevölkerungsentwicklung stärker berücksichtig werden, um auch zukünftig flächendeckend die augenärztliche Versorgung zu gewährleisten.

\section{Fazit}

Ursprünglich wurde die Bedarfsplanung eingeführt, um die Anzahl niedergelassener Vertragsärzte zu begrenzen. Dieses Ziel wurde teilweise erreicht. Auch wenn die Vertragsarztzahlen weiter angestiegen sind, ist der Anstieg geringer 
als vor der Einführung der Bedarfsplanung. Auch die räumliche Verteilung der Versorgung nähert sich dem Bedarf (wie er in den Richtlinien des G-BA ausgewiesen ist) an. In der Augenheilkunde gibt es nur einen Planungsbereich, der von Unterversorgung bedroht ist. Dennoch bestehen deutliche Versorgungsunterschiede zwischen den Planungsbereichen.

Die zukünftige Versorgung ist durch einen steigenden Bedarf charakterisiert, der sich aus der Alterung der Bevölkerung in Deutschland ergibt (demografisch bedingter Nachfrageanstieg bis
2030 um 10 bis $12 \%$ ). Gleichzeitig wird vermutet, dass das zukünftige Arztangebot sinkt, weil in den nächsten 10 bis 15 Jahren ein überproportionaler Anteil an Vertragsärzten altersbedingt ausscheidet. Außerdem wird erwartet, dass die "Feminisierung der Medizin« zu sinkenden Wochenarbeitszeiten der Ärzteschaft führt. Um die Versorgung aufrecht zu erhalten, müssen die Zugänge an neuen Vertragsärzten die Abgänge an ausscheidenden Vertragsärzten übersteigen.

Sowohl die Analysen der KVN als auch die eigene Analyse, die von einer
Zunahme des Arztangebots um 6\% (Szenario 3) ausgehen, lassen aber zumindest bis 2020 erwarten, dass kein substantieller Engpass in der Augenheilkunde auftritt. Die Aufhebung der Altersgrenze von 65 Lebensjahren für Vertragsärzte scheint sich positiv auf das Arztangebot auszuwirken. Um Unterversorgung in weniger attraktiven Planungsbereichen zu verhindern, müssen Strategien entwickelt werden, wie unterversorgte Planungsbereiche prioritär besetzt und inwieweit augenärztliche Leistungen substituiert werden können.

\section{Literatur}

Amelung V. E.; Bucholtz N.; Brümmer A.; Krauth C. (2012): Sehen im Alter, Versorgungsstrukturen und -herausforderungen in der Augenheilkunde, Medizinisch Wissenschaftliche Verlagsgesellschaft, Berlin.

BARMER GEK Arztreport (2010): Schwerpunkt: Erkrankungen und zukünftige Ausgaben, unter: http://www.svr-gesundheit.de/Gutachten/Uebersicht/GA2009-LF.pdf (abgerufen am 27.09.2011)

\section{Bundesärztekammer (2000 2009): Die} ärztliche Versorgung in der Bundesrepublik Deutschland, unter: http://www.bundesaerz tekammer.de/page.asp?his=0.3.8175 (abgerufen am 18.09.2011)

Deutscher Bundestag (2011): Über- und unterversorgte ärztliche Planungsbereiche in Verbindung mit der Relation Ärzte/Ärztinnen zur Bevölkerung, unter: http://dipbt.bundestag de/dip21/btd/17/066/1706632.pdf (abgerufen am 12.11.2011)

Finger R.P.; Fenwick E.; Marella M.; Dirani M.; Holz F.G.; Chiang P.P.C.; Lamoureux E.L. (2011a): The Impact of Vision Impairment on Vision-Specific Quality of Life in Germany. Investigative Ophthalmology \& Visual Science. doi:10.1167/iovs.10-7127

Finger R.P.; Fimmers R.; Holz F.G.; Scholl H.P.N. (2011b): Incidence of blindness and severe visual impairment in Germany - projections for 2030. Investigative Ophthalmology \& Visual Science. doi:10.1167/iovs.10-6987

Fritz-Beske-Institut (2009): Das Gesundheitswesen auf eine älternde Bevölkerung einstellen - Neue Daten zeigen dringenden Behandlungsbedarf auf, unter: http://www. aok-bv.de/imperia/md/aokbv/politik/reformaktuell/114 pm lang-msp.doc.pdf (abgerufen am 12.11.2011)
Gemeinsamer Bundesausschuss (2011): Richtlinie des Gemeinsamen Bundesausschusses über die Bedarfsplanung sowie Maßstäbe zur Feststellung von Überversorgung und Unterversorgung in der vertragsärztlichen Versorgung (Bedarfsplanungs-Richtlinie). Bundesanzeiger 2011: 2768

Hess R. (2011): Reformbedarf der ambulanten Bedarfsplanung. In: Gesundheit und Sozialpolitik 1/2011: 21-25

Kassenärztliche Bundesvereinigung (2011): Daten zur augenärztlichen Versorgung in Deutschland. (auf Anfrage zur Verfügung gestellt am 16.09.2011)

Kassenärztliche Bundesvereinigung (2008): Die Bedarfsplanung des ambulanten Sektors in Deutschland und die Notwendigkeit ihrer Weiterentwicklung. Bericht. Berlin.

\section{Kassenärztliche Vereinigung Niedersachsen} (2007): Prognose der Entwicklung der Arztzahlen für das Jahr 2020. Hannover.

Kopetsch T. (2011): Bedarfsplanung - Das Reformkonzept der Kassenärztlichen Bundes vereinigung. In: Gesundheit und Sozialpolitik 1/2011: 34-42.

Mühlbacher A.C.; Wessels M. (2011): Neue Wege in der Bedarfsplanung in Deutschland: Haben wir ein Mengen- oder ein Verteilungsbzw. Allokationsproblem? In: Gesundheit und Sozialpolitik 1/2011: 53-63.

Resnikoff S.; Pascolini D.; Etya'ale D.; Kocur I. Pararajasegaram R.; Pokharel G.P.; Mariotti S.P. (2004): Global data on visual impairment in the year 2002. Bulletin of the World Health Organization, 82(11), 844-851. doi:/SoO4296862004001100009
Schallock M.; Czihal T.; Graf von Stillfried D. (2009): Zukünftige vertragsärztliche Versorgung in dünn besiedelten ländlichen Räumen Thüringens: Eine kleinräumige Analyse zum Jahr 2020, im Auftrag des Zentralinstituts für die kassenärztliche Versorgung, Berlin

Statistisches Bundesamt (2009): Bevölkerung Deutschlands bis 2060: 12 . koordinierte Bevölkerungsvorausberechnung, unter: http:// www.destatis.de/jetspeed/portal/cms/Sites/ destatis/Internet/DE/Presse/pk/200g/Bevoelkerung/pressebroschuere_bevoelkerungsen twicklung2009, property=file.pdf (abgerufen am 12.10.2011)

Statistisches Bundesamt (2010): Mikrozensus. Erwerbstätige nach Berufsgruppen, ausgewählten Berufsordnungen und normalerweise je Woche geleisteten Arbeitsstunden. In: Fachserie 1 Reihe 4.1.2. Wiesbaden.

Statistisches Bundesamt (2011): Bildung und Kultur. Studierende an Hochschulen. In: Fach serie 11 Reihe 4.1. Wiesbaden.

Uhlemann T.; Lehmann K. (2011): Reformkonzept des GKV-Spitzenverbandes zur Weiterentwicklung der Bedarfsplanung. In: Gesundheits- und Sozialpolitik 1/2011: 26-33.

World Health Organization (WHO) (2004): Fact Sheet No. 282. Magnitude and causes of visual impairment, unter: http://gat4provip. eu/media/upload/C5-fact_sheet-WHO_document[1].pdf (abgerufen am o8.11.2011)

Wille E.; Erdmann D. (2010): Gesundheitsökonomischer Stellenwert flächendeckender Facharztversorgung. Gutachten im Auftrag des Deutschen Facharztverbandes / Potsdamer Runde e. V. 